\title{
Research on the method of processing of legal language by the information technology
}

\author{
Li Ma \\ School of Foreign Languages, East China University of Political Science and Law,Shanghai, China \\ LiMa@163.com
}

Keywords: Metacognitive reading strategies, Legal language, Information processing.

\begin{abstract}
Metacognition refers to the series of skills in self- construction and adjustment in all cognitive stages. Successful learners have acquired many metacognitive strategies to meet requirements in different learning contexts. Metacognitive reading strategies play a positive role in the process of reading such as controlling the information flow, monitoring and supervising the process of cognition. With the presentation of the cognitive theory, the reading models and the strategies applied in second language reading comprehension on the basis of cognitive theory, several approaches in information processing of second language legal language texts are explored. Hence the inspiration in language teaching are drawn with the employment of metacognitive reading strategies.
\end{abstract}

\section{Metacognition and monitoring strategies}

Metacognition is defined as the knowledge and the regulation of human cognition, the self-constructed and adjustment skills infiltrated in the various cognitive domains. It is also referred to as " the thinking of one's own thinking"[1] . Research on metacognitive ability can be classified into four categories. The first category is the study of cognitive monitoring ,exploring human understanding of their own knowledge and thinking processes as well as the accuracy of their current monitoring situation for their own knowledge[2]. The second area of study focuses on the adjustment of human thinking process for the different needs in different scenarios[2]. The third category of metacognitive research is to investigate how people choose to adjust their strategies based on the information one needs to monitor.

Modern psychology holds the view that metacognition is a advanced element in intelligence structure and it is a higer level of thinking monitoring cognitive process actively in learning. These monitoring behavior is composed of proposing the learning task, monitoring the process of understanding, as well as evaluating the process of task comletion. In language teaching, metacognitive monitoring is an important part of the cognitive ability study, with the focus on human knowledge and understanding of their own thingking processes,as well as the accuracy of their own knowledge monitoring.

\section{The application of monitoring strategies in the legal text information processing}

Directional monitoring. Directional monitoring refers to having a clear reading purposes and an intented reading strategy, such as scanning, skimming and peruse. Scanning is to determine the information and get the main idea quickly, so as to find the answers to a quesiton and the solutions to a problem.Skimming applies to the longer materials, mainly for the purpose of overall understanding. Perusing is applied in the reading of a short material, usually for the detailed information,with the use of $\mathrm{W}$ - questions to understand the context of the subject and the plot development.

In the legal text information processing, scanning ,skimming and peruse can all be applied to grasp the main idea of the text in regard to the highly stylized features of legal discourse structure. Legal written texts are composed of legislative texts (such as laws and regulations), judicial documents (such as court records), language materials, suspicious texts and so on. With comparative studies, we can see that the similar discourse structure in both Chinese and English 
legislative texts leads to the similarity between the legal discourse of the two languages,sharing the discourse structure of transferring from descriptive component to provisional component, from the decree and / or preface to the specific provisions. The structural levels are distinct and are dveloped from macro to micro, from subjects / general to provisions, from important provisions to minor provisions Therefore, the information processing at this stage is focused on the main ideas and finding the required tasks.

Process Monitoring. Process monitoring means thinking while reading, identifying the important information that the reading materials suggest, monitoring the depth of reading with the purpose of obtaining the detailed information in the text. Other approaches as guessing can be employed to grasp the meaning in the context to fulfill the relevant reading requirements.Before the reading begins, the teacher can also design some guiding questions to give the students a specific goal or task to achieve the reading process monitoring.

There are many synonyms or alternative terms in legal English terminology,in which special attention is needed in reading. Given the rigor and accuracy of legal terminology in English, they can not just replace each othe in many circumstances. For example, brand and trademark are used as the names of the business, goods or services, but they are not synonymous (although some people put them all as "shangbiao"). The difference is that brand (Pinpan) mainly refers to the pre-established, famous trade names used in advertising.It is not a formal legal term and generally has no exclusive rights. While "trademark(shangbiao)" is a typical legal term for the registration of the trade mark with the exclusive right to use . It is an infringement if used without consent. They can be words, symbols, etc. The manufacturers or merchants use "trademark" to distinguish their commodities from others. As can be seen, with the rich sematic meaning of English, the same word in a different language domains represents different concepts. One should first have a clear understanding of the source and characteristics of English legal terminology when using metacognitive reading strategies in process monitoring.

The sources of English legal terms can roughly be classified into three categories: the first category is the modern common formal language, which constitutes the mainstream of legal English; the second category is the less- used or out -of-use Middle-age English; the last category is the borrowed words like Latin and French. English legal terminology is characterised by the following main features:

(1) From general to specific. A term referring to the different similar objects can be used to indicate any of them. If this referrence is common in the language, the term acquires a newlyspecified meaning. For example:, in "He got life (He was sentenced to life imprisonment) ", "life" here refers specifically to "life imprisonment".

(2) From common nouns to proper nouns: The term "party" originally refers to "social gathering, a political organization". But in legal English, it means " one of the people involved in a legal agreement or dispute." "Instance", originally means” an example in real life situation”, but "second instance" means specifically to "the second trial" in legal context. The generalizing process of the special terms can be seen as the process of giving means to the meaningless terms. Another example is the term "minor" with the original meaning of "secondary,trivial," but it's legal semantic meaning is "young person under a certain age." Some legal terminologyies are extended general terms, such as "bench" which refers to the judge or court. "Bar" refers to a "public house" as a general term, but " legal profession" as a legal term.Therefore, we should understand the specific meaning of the term by its legal concept. The meaning must be determined from the context so as to understand the meaning of the original text accurately.

Slight Monitoring. Slight monitoring is used in self-monitoring questions, testing if one's own answer is correct or not, reasoning with multi-angle analysis and getting to know how to use effective strategies to deal with general issues. After the completion of reading tasks, the teacher can help students evaluate their own understanding, enable students to assess the results of their cognitive activity and promptly correct their error. and thus improve their reading proficiency. Slight monitoring is a strategy guiding students to have self-monitoring and self-evaluation, so they can promptly evaluate on their cognitive activities in view of their cognitive objectives. With the 
results and feedback, they can correctly estimate their goals and evaluate the effectiveness of various cognitive strategies with continuous monitoring and adjustment so as to achieve the intended goal.

To strengthen the students metacognitive awareness, two areas can be addressed in evaluating the students' reading progress: (1) Is there a theoretical system in the text and an intrinsically logical connection in the words and structures?

In process monitoring, the reader has to understand the source and the key features of the English legal terminology. But in the self-monitoring process of slight monitoring strategy, we have to attach importance to the logical connection between legal terms and the entire text as well as the specific legal connotation of the term. Legal terminology can be broadly divided into two types: one is the unique legal terminology, which only occur in the legal documents. A case in example is the term "thalweg" in international law, which refers to the dividing line in the middle of the river between countries, namely, the centerline of the river channel. The other one is not the unique terminology in legal language. It may be used in other genres but has a specific meaning in legal documents. Example in legal context are like "action"-- "to prosecute"; "cost"-- "litigation costs" , "declaration"-- "confirmation".

Are there language phenomena reflecting different pragmatic features in the text? The so-called pragmatic feature refers to the semantic meaning related to the culture, history and customs added to the conceptual meaning of the language. It is difficult to have a complete understanding of the meaning of language without the knowledge of the ethnic and cultural background. Pragmatic features are prevalent in language and the understanding of law is no exception. Legal terminoloy is the reflection of the legal culture as well as the historical origin of a country. With the hundreds of years of development in human legal history, it is inevitable for the legal terms to have the "legal pragmatic features" reflecting the characteristics of the legal culture. Special cultural connotations in legal terms are common in English, especially in laws or regulations and they can not be ignored in reading [4]. In Chinese laws or regulations promulgated in prohibiting or preventing various crimes or wrongdoings, terms like "punish (chengzhi) ", "prevent (fangzhi) " and "anti (fan)", "sin (zui) " . Examples are "The Methods for Prohibiting the Entry and Exit of Currency in People's Republic of China", "The Regulations for Punishing Counter-revolutionaries in People's Republic of China”, etc. The terms like "count, against, anti, preventive, forbidding, offence or crime” are not commonly used in the laws or regulations in Britain or America. In understanding the English law Theft Act, the translator should know that the term "theft" is controdictory from its meaning in this unique legal context ,and it should be understood as anti-theft. Thus "Theft Act" should be translated as "Anti-theft law" or "Theft Crime Law" instead of "Theft Act." Otherwise, it could be understood as " theft is lawful and should be specially formulated by laws to regulate it" . Similarly, "Dumping Law" indicates "Anti-dumping Law" in Chinese understanding.[7].There have been a lot of controversies for the understandings of the term " gongsi (company)". Some hold the view that " corporation" is more formal and of a larger scale than "company", but the latter is more commonly used. The influential Chinese "English Law Dictionary" translated both " foreign company" and "foreign corporation" into "waiguo gongsi (foreign company)" [5]. In fact, the Chinese companies are classified basically in compliance with the form of the civil law countries, which is very different from Britain and America. In English, corporation and company are polysemy. Besides corporation, the term "Company" can also mean "legal persons". When it refers to "company", it is equivalent to "business corporation" or "for- profit corporation.”In addition, "Company" also means "firm" which has no legal personality. It is generally used in British English. Both terms are used in Australian English without any differences. U.S. companies are generally divided into close corporation and publicly held corporation; UK generally divides them into privately limited company and publicly limited company; while Australia generally divides companies into public company and proprietary company. Therefore, it is hard to tell which is more commonly or more formally used than the other or which term represents the company with the bigger size. Meanwhile as the company classification in Anglo-American countries is different from civil law countries (including China), legal implications of PLC are different if we use it to 
represent limited liability company because of the substantial differences. Furthermore, as each state in the United States has its own corporate law, any corporations that are not established in accordance with the corporate law of the state are called "foreign corporations", which is translated as "waizhou gongsi(out of state company)" in Chinese while foreign companies are called "alien corporations."

\section{Conclusion}

In short, the evaluation is the ability to understand the characteristics and make personal reading material analysis. After completing the learning task of reading material, a good learner will reflect on the completion of the process of reading tasks. This process gives the reader a clear plan to check the situation and the role learning strategies plays in reading. The higher the language proficency, the more metacognitive strategies are needed. This indicates that successful language learners are better at using metacognitive strategies. They are capable to use and monitor strategies more effectively [3]. Besides, teachers should design the reading program integrating knowledge in the matarial and the metacognitive strategies, creating the opportunities for the students to learn and experience, promoting and teaching metacognitive strategies, guiding students to have a better understanding of the material and apply the strategies to improve their reading comprehension.

Metacognitive theory maintiains that students' learning ability constitutes a variety of cognitive abilities, including the meta-cognitive ability of self-learning. The cognitive level and the validity of cognitive process depends largely on the learners' meta- cognitive level. The students' meta-cognitive development plays an important role in their language learning potentials. An important way to improve learning or metacognitive abilities is to implement metacognitive strategies, which is an operational process for the students to think in their learning activities with the purpose of teaching the students how to learn. Thus, teachers should actively make use of metacognitive strategies in college English teaching, so that the students can learn about their learning ability and characteristics. They can improve learning efficiency by using the appropriate metacognitive strategies. Once the students meta-cognitive skills are trained and developed, they can learn how to choose the appropriate ways in learning, regulate their own learning, and eventually improve their overall learning ability.

\section{Acknowledgement}

This research is funded by 2012 Chinese Social Sciences Fund (12BYY019).

\section{References}

[1] Hacker, Douglas J.Definitions and Empirical Foundations. In Hacker, D. J., Dunlosky, J.\& A. Graesser (Eds.), Metacognition in Educational Theory and Practice. Mahwah, NJ: Lawrence Erlbaum Associates.1998:1-24.

[2] Kluwe, R. H.Cognitive knowledge and executive control: Metacognition. In D. R. Griffin (Ed.),Animal mind - human mind.New York: Springer-Verlag.1982: 201-224

[3] "Chinese-English Dictionary" (revised edition). Beijing: Foreign Language Teaching and Research Press, 1995.

[4] "Modern Advanced Learner's Dictionary". Oxford University Press, 1978.

[5] "English Law Dictionary", Beijing: Law Press, 2005.

[6] F.S. Yuan, D.F. Xiao. Metacognitive strategies in TEM4 and its relationship with reading achievement Department. Foreign Languages Teaching, 2006 (4).

[7] L. Song, and Cheng Rukan. 2006. On the Cultural Issues of Legal Terms Translation. Journal of Southwest University of Political Science and Law.(6):22 